\title{
Bowel Ultrasound for Acute Diverticulitis - Technical Aspects in Three Different Clinical Settings
}

\author{
Anda Les ${ }^{1,2}$, Ion Bancila ${ }^{1}$, Anca Dimitriu ${ }^{1,2}$, Bogdan Cotruta ${ }^{1}$, Iuliana Pirvulescu ${ }^{1}$, Ioana Lupescu ${ }^{2,3}$, \\ Razvan lacob ${ }^{1,2^{*}}$, Cristian Gheorghe ${ }^{1,2}$
}

'Digestive Diseases and Liver Transplantation Center, Fundeni Clinical Institute, Bucharest 2"Carol Davila" University of Medicine and Pharmacy, Bucharest, Romania

${ }^{3}$ Radiology and Imaging Department, Fundeni Clinical Institute, Bucharest, Romania

\section{ABSTRACT}

Acute diverticulitis is a frequent complication in patients with colonic diverticulosis. The diagnosis is based on the clinical presentation, biological markers and imaging. Abdominal ultrasonography is, in many centers, the first examination in patients presenting with abdominal pain. Bowel ultrasonography has the advantage of being an inexpensive, nonionizing, readily available and repeatable examining method, but needs an experienced operator, and it is, thus, not widely used in clinical practice. We present a case series of acute diverticulitis, using bowel ultrasonography to establish the diagnosis in three different clinical settings: uncomplicated diverticulitis, abscess complicated diverticulitis and neoplasia associated diverticulitis. The patients were examined at admission, abdominal pain being the main symptom. The ultrasound examination started with a 3-5 $\mathrm{MHz}$ probe as in the case of classic ultrasound, followed by a $5-11 \mathrm{MHz}$ probe examination that allowed adequate investigation of the bowel loops and establishing a diagnosis of acute diverticulitis based on ultrasonographic criteria. All patients had the diagnosis confirmed by a computer tomography scan and subsequently underwent antibiotic treatment. All patients had ultrasonographic characteristics suggesting parietal inflammation, overlapping with the lesions observed at CT scan which were indicative of acute diverticulitis. Both imaging techniques were able to show complications and extraintestinal alterations. Abdominal ultrasound is the imaging method most frequently used in patients presenting with abdominal pain. By using the appropriate transducer, acute diverticulitis and complications could be accurately diagnosed.

Key words: diverticular disease, diverticulitis, bowel ultrasonography

\section{INTRODUCTION}

Diverticulosis is a common condition affecting particularly patients over the age of 40 years and its incidence increases with age. Diverticulitis represents the acute inflammation of a diverticula and appears in about $20 \%$ of patients with diverticulosis (1).

The diagnosis can often be suggested on clinical grounds, but because the differential diagnosis is wide, many authors recommend radiologic assessment (2). Ultrasound is one of the most frequently used tools in the setting of acute

\author{
*Corresponding author: \\ Razvan lacob MD, PhD \\ Digestive Diseases and Liver \\ Transplantation Center \\ Fundeni Clinical Institute \\ Bucharest, Romania \\ 258 Fundeni Str, sector 2 \\ E-mail: raziacob@gmail.com
}

Received: 18.03 .2021

Accepted: 22.05.2021 
abdomen. Bowel ultrasonography is useful both in detecting early alterations of the bowel wall, suggesting diverticulitis, and local complications (1). The limitation of bowel ultrasonography (US) consists in the pelvic location of diverticulitis, where the ultrasound cannot reach, in which situation computed tomography (CT) is superior (1). We report a case series of patients with acute diverticulitis diagnosed by bowel ultrasonography, in three different clinical settings: uncomplicated diverticulitis, abscess complicated diverticulitis and neoplasia associated diverticulitis.

\section{CASE REPORT}

The study case series included three patients that were evaluated by ultrasound for abdominal pain and a diagnosis of acute diverticulitis was established based on the observed bowel wall alterations. The diagnosis was confirmed by a CT scan and bowel ultrasonography was used for follow-up. The bowel pathological findings were recorded using both imaging techniques.

The first patient is a 62-year-old man who presented to the hospital complaining of abdominal left flank pain, irradiating to the left hip and scrotum. The pain started $48 \mathrm{~h}$ before presentation and was associated with fever $\left(38.5^{\circ} \mathrm{C}\right)$. He did not report rectal bleeding, vomiting or nausea, or urinary symptoms. The patient had been recently diagnosed ( 6 months before) at colonoscopy with diverticulosis of the sigmoid colon.

The physical examination showed no particularities other than a tender left flank on palpation. Blood tests revealed moderate leukocytosis with neutrophilia and a high CRP. Pneumoperitoneum or bowel obstruction were excluded at plain abdominal X-Ray.

An abdominal ultrasound was performed in the emergency room at admission and a diagnosis of acute diverticulitis was suggested; the following day, the patient was examined by CT scan, confirming the diagnostic suspicion.

The patient was started on standard therapy for acute diverticulitis (bowel rest, parenteral hydration, third generation cephalosporin, Metronidazole and Rifaximin as antibiotics). He recovered well during the 7 days of hospitalization, becoming asymptomatic and reaching normal values of the blood tests. At discharge, a scheduled colonoscopy was recommended as well as continuation of $800 \mathrm{mg}$ Rifaximin per day for another 7 days and monthly repeated administrations for 10 days, as studies have showed this approach improves symptoms and recurrence risk (3).

6 months after the acute episode, the patient did not relapse and ultrasonographically no alterations were observed. The continuation of a high fiber diet was recommended.

The second case is of a 67-year-old female who suffered from left iliac fossa pain persisting for 3 days prior the presentation in our clinic. She had no complaints of nausea, vomiting, diarrhea or loss of appetite. She has a medical history of diabetes mellitus, hypertension and hyperlipidemia and a surgical history of cholecystectomy for uncomplicated acute cholecystitis. The physical examination revealed a tender abdomen in the left iliac fossa and laboratory measurements showed leukocytosis and high C-reactive protein.

An abdominal ultrasound was performed and pathological findings suggestive for complicated acute diverticulitis were observed.

A CT scan confirmed the diagnosis of sigmoid diverticulitis and the presence of an abscess. The patient was started on intravenous antibiotics (Metronidazole and Ciprofloxacin); after 10 days of treatment, she was reevaluated using bowel ultrasonography and no collections were noticed. The patient was discharged the next day with no symptoms.

3 months after the diagnosis of acute diverticulitis, the patient was asymptomatic and was reevaluated using bowel ultrasonography. No collections were observed but alterations of the bowel wall and densification of the mesentery persisted. She refused to undergo scheduled colonoscopy. High fiber diet was recommended.

The third patient was a 79-year-old male admitted with a 7-days history of sudden onset epigastric abdominal pain. He also reported 4 loose stools per day during the last week. His medical history revealed atrial fibrillation, hypertension and a history of colon diverticulosis with several episodes of acute diverticulitis, the last being 12 months prior to admission. The patient had a complete colonoscopy 5 years prior to this episode. He reported no past abdominal surgical procedures. Clinical examination was unremarkable and laboratory tests revealed an iron deficiency anemia and a slightly elevated C-reactive protein.

Abdominal ultrasonography was performed and an abnormal transverse colon wall was found. Acute diverticulitis was suspected and a CT scan was performed the following day, confirming the diagnosis but suggesting also associated neoplastic pathology. Conservative treatment was started with intravenous antibiotics and infusions and after a short period of bowel rest, he recovered and was discharged after 7 days. On colonoscopy at 2 weeks post-treatment, malignancy of the transverse colon was confirmed and surgery was scheduled. 


\section{Ultrasonographic technique}

Patients were ultrasonographically assessed by a single experienced examiner using 2 ultrasound machines (Samsung Medison and Aloka Arietta). The investigator was informed about patients' symptoms and medical history.

The ultrasonographic examination was performed with the patient in supine position, without special preparation before. Initial examination was carried on with a 3-5 $\mathrm{MHz}$ convex probe, followed by a $5-11 \mathrm{MHz}$ micro-convex transducer. The convex probe was used to establish the area of interest guided by the patient's symptoms and the micro-convex probe was utilized to distinguish details of the bowel wall and extraintestinal alterations, thus facilitating the diagnosis.

For each subject, sonographic measurements of bowel wall thickness were noted and alterations of the bowel wall were described. Extraintestinal pathological findings such as mesenteric hypertrophy, free abdominal fluid or the presence of an abscess were documented.

\section{Results of bowel ultrasound examination}

The imagistic findings for each patient are summarized in table 1.

In the first case, the area of interest was cephalad to the iliac vessels and to the psoas muscle where an intestinal lumen corresponding to the sigmoid colon showed signs of inflammation (thickened bowel wall, with mesenteric hypertrophy) and a hypoechoic extension to the bowel wall, with a hyperechoic area corresponding to an inflamed diverticulum. Figure 1 shows ultrasonography (US) images of the inflamed

Table 1. Pathological findings in imaging methods (BUS and corresponding CT scan)

\begin{tabular}{lccc}
\hline & Patient 1 & Patient 2 & Patient 3 \\
\hline Bowel wall thickness-US & $5 \mathrm{~mm}$ & $7 \mathrm{~mm}$ & $9 \mathrm{~mm}$ \\
\hline Bowel wall thickness-CT & $5 \mathrm{~mm}$ & $6 \mathrm{~mm}$ & $9 \mathrm{~mm}$ \\
\hline Doppler signal & moderate & important & important \\
\hline Length of pathological loop- US & $5 \mathrm{~cm}$ & $5 \mathrm{~cm}$ & $10 \mathrm{~cm}$ \\
\hline Length of pathological loop- CT & Not assessed & Not assessed & $10 \mathrm{~cm}$ \\
\hline Abscess seen in US & no & yes & no \\
\hline Abscess seen in CT & yes & yes & no \\
\hline Stranding of the pericolic fat- US & yes & yes & yes \\
\hline Stranding of the pericolic fat- CT & yes & yes & yes \\
\hline Lymph nodes- US & no & no & yes \\
\hline Lymph nodes- CT & no & yes & yes \\
\hline Presence of diverticula- US & yes & yes & yes \\
\hline Presence of diverticula- CT & yes & yes & yes \\
\hline
\end{tabular}

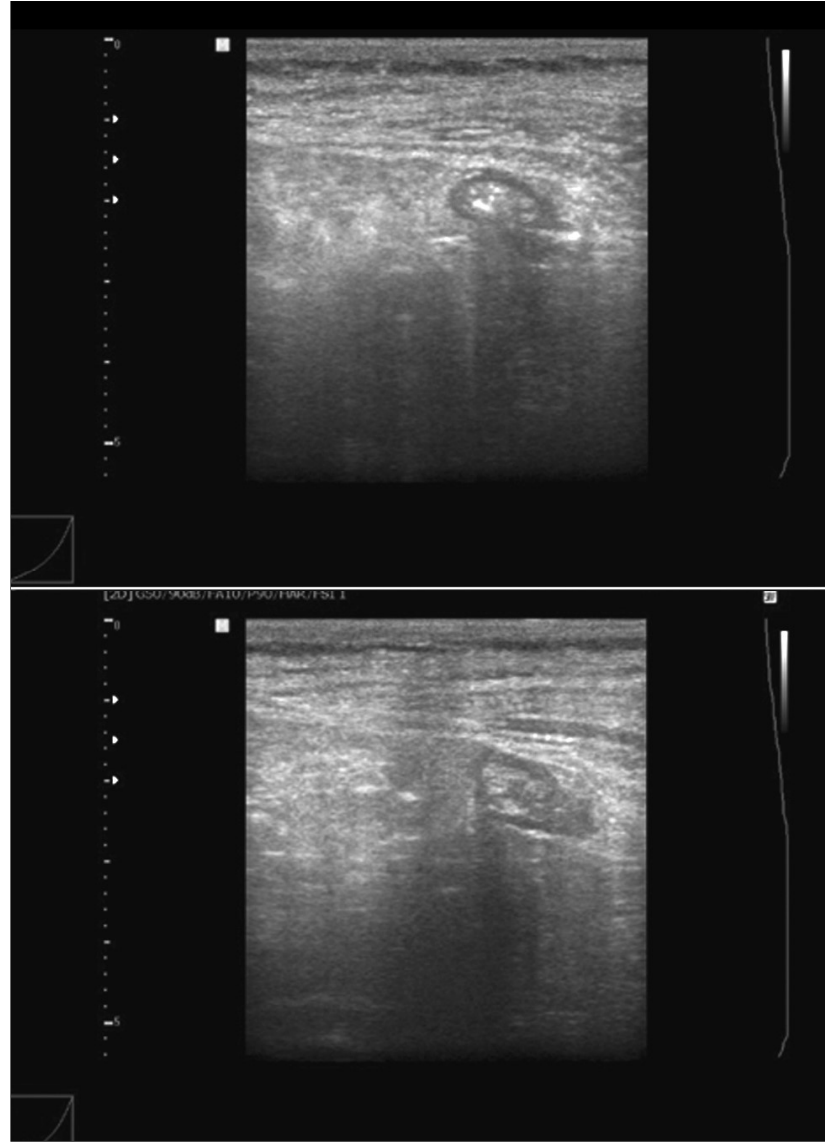

Figure 1 - BUS transverse section of an intestinal lumen above the left iliac vessels corresponding to the sigmoid colon with signs of early diverticulitis: thickened bowel wall, hypoechogenic wall suggesting inflammation and a hyperechogenic spot surrounded by a hypoechogenic rim suggesting a diverticulum

bowel wall. The abdominal computer tomography (CT) scan on the following day confirmed the ultrasonographic diagnosis of diverticulitis. Large diverticula (10 $\mathrm{mm}$ ) were noticed at the level of the sigmoid and descending colon, with inflammation of the adjacent bowel wall, creeping fat surrounding it and a small fluid accumulation. Figure 2 shows the CT findings in this case.

In the second patient, abdominal ultrasonography identified a pathological sigmoid colon corresponding to the area of the maximum abdominal pain. An increased bowel wall thickness was observed, with altered stratified structure and a 4-centimeter hypoechoic area with no color Doppler signal was noticed next to the sigmoid colon, surrounded by hyperechoic mesenteric fat suggesting pericolic abscess. The descending colon was examined, and a hypertrophic muscular layer was noticed, hinting to the presence of colon diverticula. Figure 3 shows US images of the inflamed bowel wall.

The bowel ultrasound examination of our last case 


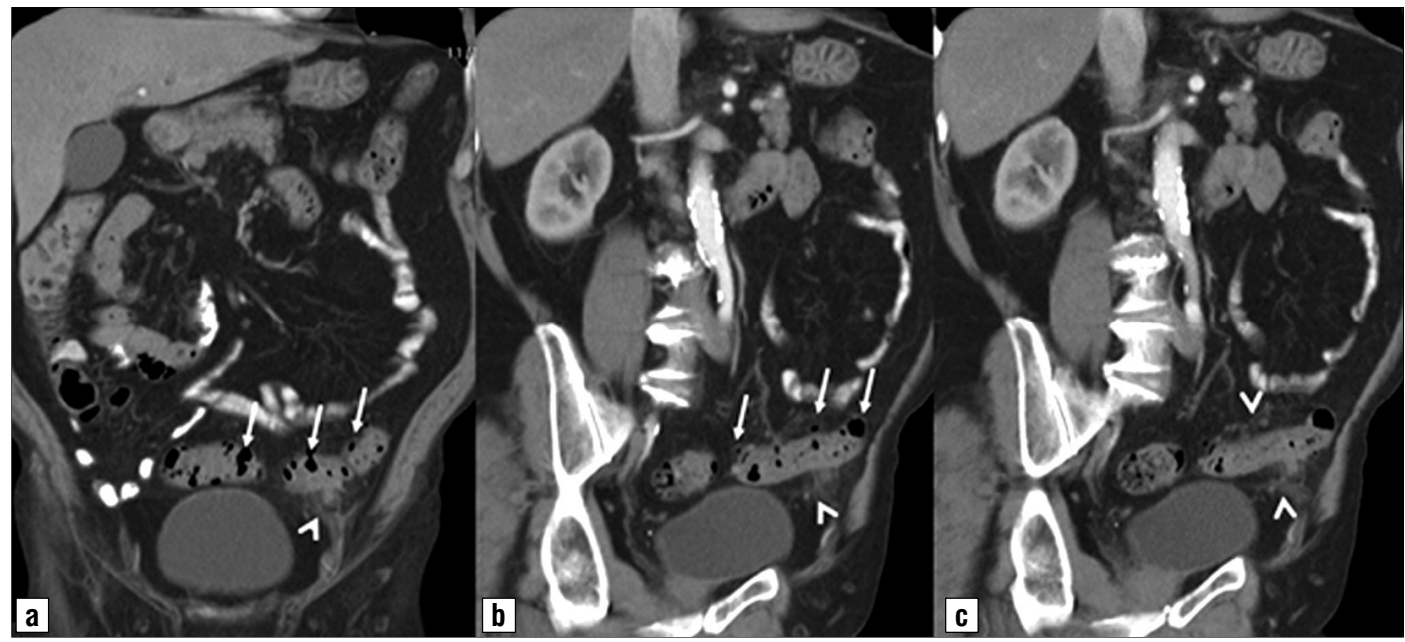

Figure 2 - MSCT evaluation in coronal (a) and coronal oblique plane (b, c): diverticulitis of the sigmoid colon, with wall thickening, multiple small diverticula (white arrow), and stranding of the pericolic fat (arrow head)

visualized an abnormal transverse colon located in the epigastrium. Multiple diverticula were noticed as hyperechoic areas that protrude from the thickened bowel wall with a hypertrophic muscular layer. The bowel became severely thickened in the right hypochondrium at the level of the hepatic flexure. The thickened segment was asymmetric, had a hypoechoic wall structure and important color Doppler flow suggesting active inflammation. A few lymph-nodes were seen proximally to the pathological loop and creeping fat around the bowel segment. Figure 4 shows US images of the pathological findings.

From our experience, bowel ultrasonography can identify parietal alterations that correspond to the diagnosis of acute diverticulitis. The ultrasound findings were similar to those obtained by CT-scan in the cases

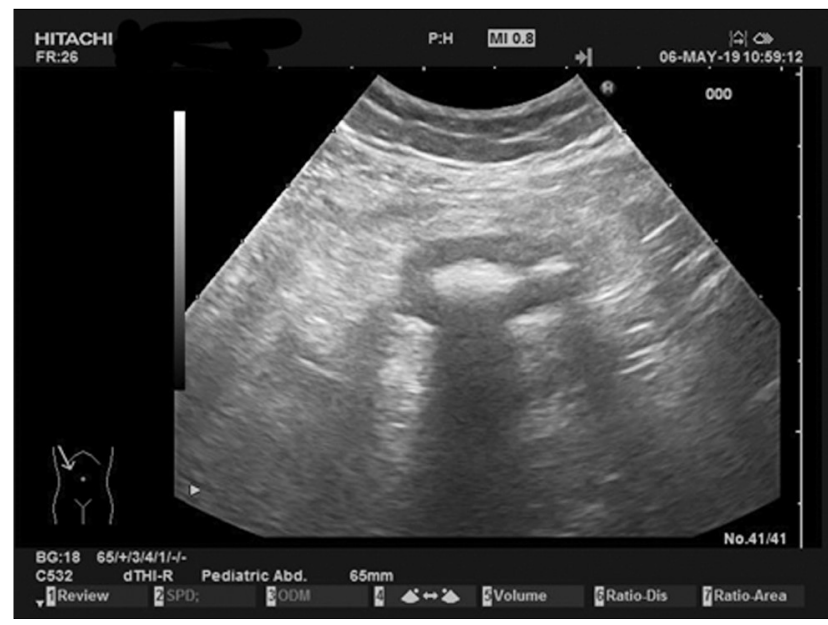

Figure 3 - BUS transverse section of the sigmoid colon with signs of acute diverticulitis: thickened bowel wall, hypoechogenic wall suggesting inflammation and surrounding hypertrophic mesenteric fat we presented and the method was used systematically in order to monitor treatment response.

\section{Discussion of technical aspects}

Bowel ultrasonography for diverticulitis has gained more attention since 1985, when Parulekar first described the alterations seen on ultrasonography in 16 patients with this disease (4). Given the higher risk of perforation, colonoscopy is contraindicated in acute diverticulitis. As opposed to ultrasonography, CT is an irradiating diagnostic tool and may be unavailable in the short term.

Bowel ultrasonography has the advantage of being an inexpensive examination method and does not use ionizing radiation. US is a real-time examination 
allowing the examiner to evaluate the motility and blood flow of the bowel loops, and to correlate the findings with the area of maximal pain (5).

The technique of bowel US consists first in an evaluation with a curvilinear 3,5-5 MHz transducer and, if depicting an abnormality, switching to the linear or curved high frequency probe $(7,5-13 \mathrm{MHz})$, to have a more detailed image. If the patient can localize the pain, the examination can begin there. Ideally, the patients need 4-5 hours of fasting before the examination, but this is not mandatory (6).

To perform an efficient scanning, graded compression is needed. This allows better visualization because it displaces other bowel loops, it compresses the air and the overlying tissues. Moreover, the compressibility or rigidity of normal and abnormal bowel loops and mesenteric fat can be assessed (7).

The average normal thickness of the bowel wall is 2-4 $\mathrm{mm}$ (8). The five layers of the normal bowel wall are seen using the high frequency transducer which approximates the histologic structure (9). Physiologic bowel wall vascularization cannot be seen by US or using color or power Doppler techniques thus the identification of blood flow indicates pathologic perfusion (10).

Bowel US has an overall sensitivity of $76 \%$ in detecting bowel lesions, whereas the positive predictive value is around $98 \%$, but the negative predictive value for bowel disorders is around $58 \%$. The overall specificity of bowel diseases is $95 \%$ (11).

At US, diverticulosis is diagnosed by identifying a hyperechogenic area out of the bowel wall, with acoustic shadowing and a thinned diverticular wall, without the typical stratification pattern, due to the absence of the muscularis propria (10). Moreover, the outer hypoechoic layer corresponding to the muscularis mucosa can be markedly thickened, due to hypertrophy (12).

Regarding acute diverticulitis, studies have reported for US a sensitivity of $84-98 \%$ and a specificity of 80-98\% (13). Ultrasound seems to be highly sensitive and specific for uncomplicated acute diverticulitis and for pericolic abscesses $(13,14)$. Sonography has comparable accuracy with $\mathrm{CT}$, but it is operator dependent and not yet widely used (15).

The staging of lesions seen in diverticulitis is similar in US and CT. In 2002, Ambrosetti et al (16) proposed a staging system, dividing the abnormalities seen on $\mathrm{CT}$ in 2 categories: moderate diverticulitis, defined by localized sigmoid wall thickening and fat stranding; and severe diverticulitis, when observed wall thickening with abscess, extraluminal air or extraluminal contrast.
The diagnostic criteria for diverticulitis used in CT imaging are the same as those used in US: at least one diverticulum with signs of inflammation or pericolic fat stranding and thickening of the bowel wall (16). Signs of diverticulitis seen ultrasonographically are: thickening of the bowel wall, a hyperechogenic area with a surrounding hypoechoic rim and inflammatory pericolic fat frequently recognized as an adjacent hyperechogenic halo, seen in at least one diverticulum (17).

Kori et al introduced the term "dome sign" defining the US characteristics of diverticulitis (localized thickened wall with a hemispheric mass protruding at the thickened colonic wall and consisting of a hypoechoic wall and a central echogenic wall) (18).

The initial sign of a developing diverticulitis is bowel wall thickening with preserved stratification. A fecolith may be seen and it could be surrounded by a hyperechogenic area, that represents inflamed mesentery. In a minority of cases a small paracolic abscess may develop, which is seen as a small irregular hypoechogenic mass proximal to the fecolith. Finding the inflamed diverticulum is eased by positioning the transducer at the area of maximum pain. The ultrasonographic alterations may persist for a long time after symptom disappearance (12).

The inflammation of the bowel wall produces an asymmetrical or circumferential hypoechoic mural thickening that may demonstrate vascular flux on Doppler scanning (10).

Ultrasonography is useful in identifying complications of diverticulitis, like micro-perforation (small air bubbles), pericolic abscess (anechoic collection that may contain pockets of air or debris) $(1,12,17)$ or fistulas. US may have an important role in diagnosing colo-vesical fistulae secondary to diverticulitis, by observing air in the urinary bladder. Trans-anal endosonography can establish the diagnosis of fistula to the vagina. Also, endo-vaginal US plays an important role in women with deeply located diverticulitis $(12,19)$.

Contrast enhanced ultrasonography (CEUS) could be useful in diverticulitis imaging. The bowel alterations are similar to classic ultrasonography: peri-diverticular inflammatory reaction, mural and peri-diverticular abscess formation, and concomitant segmental bowel wall thickening with hypervascularity (20).

Complications of diverticulitis such as pylephlebitis and abscesses can also be recognized by CEUS and, most importantly, CEUS can be used in the differential diagnosis of an inflammatory mass and abscess (21). It may also help in differentiating ischemic intestinal necrosis (lack of contrast filling) with inflammation (symmetric thickening with low resistivity index) from 
neoplasia (asymmetric thickening with high resistivity index) (22).

Regarding the stenosis that may appear as a complication of repeated attacks of diverticulitis, elastography can evaluate the stiffness of tissues by measuring their elasticity, and display it as a colored real-time elastogram in the conventional B-mode ultrasound image with a special software, thus revealing the fibrotic alterations in the colonic wall (23).

US findings should take into consideration the differential diagnosis with mild inflammatory bowel disease (24), adenocarcinoma, lymphoma, Crohn's disease, ischemic colitis, and extracolonic inflammatory conditions adjacent to the colonic wall $(13,25)$. Mesenteric hypertrophy seen in US as a hyperechogenic mass surrounding the inflamed bowel suggests an inflammatory condition. The length of the involved segment suggests an inflammatory lesion if the segment is longer than $10 \mathrm{~cm}$, thus helping differentiating diverticulitis from a neoplasm (26). Transabdominal ultrasonography is able to reveal other causes of abdominal pain such as intussusception, it is able to visualize the thickening of the colonic wall caused by infection or neoplasia, and it could help in the choice of further examination and treatment (27).

Right sided diverticulitis, although more frequent in Asian populations, may be also found in western populations and it is crucial to differentiate it from acute appendicitis. A fecolith at the base of an inflamed appendix could be mistaken with a case of cecal diverticulitis (12).

In mild to moderate diverticulitis, Mizuki et al showed that US can be successfully used in monitoring patients (28).

Although many studies showed comparative sensitivity and specificity for US and CT, other studies found that CT is superior to ultrasonography in detecting acute diverticulitis (29).

At CT, diverticula are identified as outpouchings of the colonic wall. These diverticula may contain air, positive contrast material, or fecal material (30). Signs seen on CT are: extraluminal mass compressing the bowel, extraverted contrast material, sinus tract, fistula, abscess (31). Another important role of CT imaging is the evaluation of the possibility of percutaneous drainage (32). Intravenous contrast material is routinely administered during the $\mathrm{CT}$ evaluation, helping to demonstrate the enhancement pattern of the colonic wall, to reveal diverticular abscesses and fistulas or to delineate diagnosis from other diseases that may mimic diverticulitis (33). CT with positive rectal contrast or CT cystography may help in diagnosing fistulas (34).

Because of the lifetime cumulative radiation of CT scan, MRI could be an alternative to it. It can diagnose acute diverticulitis with a sensitivity of $94 \%$ and a specificity of $88 \%$ but at a higher procedural cost (35).

In the case of perforated diverticular disease, emergency surgery is indicated. Primary resection with primary anastomosis and diverting ileostomy is the procedure of choice for perforated diverticular disease when the risk of post-operative complications is reasonable (about 40\%) (36). When long term outcomes are considered, it must be kept in mind that patients who are surgically treated with resection of the involved segment and formation of a proximal end colostomy (Hartmann's procedure) are often left with a permanent stoma (37). Elective surgery was until recently recommended to patients who suffered more than two attacks of diverticulitis, as it was thought that the risk of perforation was higher in those with relapsing disease. Newer guidelines recommend elective surgery in patients with diverticular stricture or fistula or for patients with disease refractory to medical management (persistent pain). Laparoscopic approach is recommended because of lower rates of morbidity, mortality and hospital stay.(36).

The risk of recurrence is 42 events per 1000 patients followed-up for 5 years $(95 \% \mathrm{Cl})$ and appears to be smaller in patients older than 50 years (38). After recovering from an episode of diverticulitis, the risk of an individual requiring an urgent Hartmann's procedure is 1 / 2000 patient-years of follow-up (39). Surgery does not eliminate the risk of recurrence. The greater the extend of resection, the smaller the risk of recurrence (40).

Abdominal ultrasonography is often the first imaging investigation in patients presenting with abdominal pain and, in experienced hands, it can detect an acute inflammatory process affecting the colonic wall, suggesting acute diverticulitis. But it must be kept in mind that US frequently underestimates the severity of the inflammatory process and that computed tomography is needed, especially in a complicated disease course (12).

Current guidelines recommend complete colonoscopy 6-8 weeks after symptom resolution, but the timing of the procedure should take into account comorbidities, persistent symptoms like diarrhea or abdominal pain, or patient preferences (38). Colonoscopy is recommended in order to exclude a colonic neoplasm based on the observation that a small number of colorectal cancers (15 
cases/1000 patients) and advanced adenomas (38 cases/1000 patients) are missed at CT (38).

\section{CONCLUSIONS}

The presented series of cases underlines the advantages of bowel ultrasound examination in the diagnosis of diverticular disease and its complications. Abdominal ultrasound is frequently used as the first imaging technique in patients with abdominal pain and it should not by-pass the visualization of bowel or the extraintestinal structures that could give important clues in finding the cause of pain. In experienced hands, this procedure could be the first choice, instead of abdominal $\mathrm{CT}$, especially in uncomplicated cases, thus avoiding unnecessary irradiation and costs.

\section{Conflict of interest}

\section{All author declare that they have no conflict of interest.}

\section{Ethical approval}

Approved by the Ethics Committee of Fundeni Clinical Institute (Nr Reg 745/08/01/2018).

\section{REFERENCES}

1. Ripolles T, Agramunt M, Martınez MJ, Costa S, Gomez-Abril SA, Richart J. The role of ultrasound in the diagnosis, management and evolutive prognosis of acute left-sided colonic diverticulitis: a review of 208 patients. Eur Radiol. 2003;13(12):2587-95.

2. McKee RF, Deignan RW, Krukowski ZH (1993) Radiological investigation in acute diverticulitis. Br J Surg. 1993;80(5):560-5.

3. Bianchi M, Festa V, Moretti A, A Ciaco, M Mangone, V Tornatore, et al. Meta-analysis: long-term therapy with rifaximin in the management of uncomplicated diverticular disease. Aliment Pharmacol Ther. 2011;33(8):902-10.

4. Parulekar S, Sonography of colonic diverticulitis. J Ultrasound Med 1985;4(12):659-66.

5. Mazzei MA, Cioffi Squitieri N, Guerrini S, Stabile lanora AA, Cagini L, Macarini L, et al. Sigmoid diverticulitis: US findings. Crit Ultrasound J. 2013;5 Suppl 1(Suppl 1):S5

6. Ledermann HP, Börner N, Strunk H, Bongartz G, Zollikofer C, Stuckmann G. Bowel wall thickening on transabdominal sonography. AJR Am J Roentgenol. 2000;174(1):107-17.

7. Puylaert JBCM. Ultrasonography of the acute abdomen: gastro-intestinal conditions. Radiol Clin North Am. 2003;41(6) 1227-42, vii.

8. Fleischer AC, Muhletaler CA, James AE Jr. Sonographic assessment of the bowel wall. AJR Am J Roentgenol. 1981;136(5):887-91.

9. Kimmey MB, Martin RW, Haggitt RC, Wang KY, Franklin DW, Silverstein FE: Histologic correlates of gastrointestinal ultrasound images. Gastroenterology. 1989;96(2 Pt 1):433-41.

10. Rodgers PM, Verma R, Transabdominal ultrasound for bowel evaluation. Radiol Clin North Am. 2013;51(1):133-48.

11. Hollerbach S, Geissler A, Schiegl H, Kullmann F, Lock G, Schmidt J et al, The accuracy of abdominal ultrasound in the assessment of bowel disorders. Scand J Gastroenterol. 1998;33(11):1201-8.

12. Puylaert JBCM. Ultrasound of colon diverticulitis. Dig Dis. 2012; 30(1):56-9.

13. Schwerk, WB, Schwarz, S, Rothmund, M. Sonography in acute colonic diverticulitis: A prospective study. Dis Colon Rectum. 1992; 35(11):1077-84

14. Van Randen A, Laméris W, Wouter van Es $\mathrm{H}$, van Heesewijk HPM, van Ramshorst B, Hove WT, et al. OPTIMA Study Group. A comparison of the accuracy of ultrasound and computed tomography in common diagnoses causing acute abdominal pain. Eur Radiol. 2011;21(7):1535-45

15. Lameris W, van Randen A, Bipat S, Bossuyt PM, Boermeester MA, Stoker J. Graded compression ultrasonography and computed tomography in acute colonic diverticulitis: meta-analysis of test accuracy. Eur Radiol. 2008;18(11):2498-511.

16. Ambrosetti $P$, Jenny A, Becker C, Terrier TF, Morel P. Acute left colonic diverticulitis--compared performance of computed tomography and water-soluble contrast enema: prospective evaluation of 420 patients. Dis Colon Rectum. 2000;43(10):1363-7.

17. Maconi G, Porro B. Ultrasound if the Gastrointestinal tract. Milan: Springer; 2007. p. 19-27.

18. Kori T, Nemoto M, Maeda M, Tsuzuki Y, Ando T, Sekihara M, et al. Sonographic features of acute colonic diverticulitis: The "dome sign". J Clin Ultrasound. 2000;28(7):340-6.

19. Gritzmann N, Hollerweger A, Macheiner P, Rettenbacher T. Transabdominal sonography of the gastrointestinal tract. Eur Radiol. 2002;12(7):1748-61.

20. Braden B, Ignee A, Hocke M, Palmer RM, Dietrich C. Diagnostic value and clinical utility of contrast enhanced ultrasound in intestinal diseases. Dig Liver Dis. 2010; 42(10):667-74.

21. Esteban JM, Aleixandre A, Hurtado MJ, Maldonado L, Mora FJ, Nogués E. Contrast-enhanced power Doppler ultrasound in the diagnosis and follow-up of inflammatory abdominal masses in Crohn's disease. Eur J Gastroenterol Hepatol. 2003;15(3):253-9.

22. Roccarina D, Garcovich M, Ainora ME, Caracciolo G, Ponziani F, Gasbarrini A, et al. Diagnosis of bowel diseases: the role of imaging and ultrasonography. World J Gastroenterol. 2013;19(14):2144-53.

23. Havre $\mathrm{R}$, Gilja $\mathrm{OH}$. Elastography and strain rate imaging of the gastrointestinal tract. Eur J Radiol. 2014;83(3):438-41.

24. Pradel JA, David XR, Taourel P, Djafari M, Veyrac M, Bruel JM. Sonographic assessment of the normal and abnormal bowel wall in nondiverticular ileitis and colitis. Abdom Imaging. 1997;22(2): 167-72.

25. Wilson SR, Toi A. The value of sonography in the diagnosis of acute diverticulitis of the colon. AJR Am J Roentgenol. 1990; 154(6):1199-202

26. DeStigter KK, Keating DP. Imaging update: Acute colonic diverticulitis. Clin Colon Rectal Surg. 2009;22(3):147-55.

27. Bor R, Fabian A, Szepes Z. Role of ultrasound in colorectal diseases. World J Gastroenterol. 2016:22(43):9477-9487.

28. Mizuki A, Nagata H, Tatemichi M, Kaneda S, Tsukada N, Ishii H, et al. The outpatient management of patients with acute mild-to-moderate colonic diverticulitis. Aliment Pharmacol Ther. 2005;21(7):889-97.

29. Eggesbø HB, Jacobsen T, Kolmannskog F, Bay D, Nygaard K. Diagnosis of acute left-sided colonic diverticulitis by three radiological modalities. Acta Radiol. 1998;39(3):315-21.

30. Poletti PA, Platon A, Rutschmann 0, Kinkel K, Nyikus V, Ghiorghiu $\mathrm{S}$, et al. Acute left colonic diverticulitis: can $\mathrm{CT}$ findings be used to predict recurrence? AJR Am J Roentgenol. 2004;182(5):1159-65.

31. Doringer E. Computerized tomography of colonic diverticulitis. Crit Rev Diagn Imaging. 1992;33(5):421-35.

32. Brandt D, Gervaz $P$, Durmishi $Y$, Platon A, Morel P, Poletti PA. Percutaneous CT scan-guided drainage vs. antibiotherapy alone for Hinchey II diverticulitis: a case-control study. Dis Colon Rectum. 
2006;49(10):1533-8.

33. Horton KM, Corl FM, Fishman EK. CT evaluation of the colon: inflammatory disease. Radiographics. 2000;20(2):399-418.

34. Yu NC, Raman S, Patel M, Barbaric Z. Fistulas of the genitourinary tract: a radiologic review. Radiographics. 2004;24(5):1331-52.

35. Heverhagen JT, Sitter H, Zielke A, Klose KJ. Prospective evaluation of the value of magnetic resonance imaging in suspected acute sigmoid diverticulitis. Dis Colon Rectum. 2008;51(12):1810-5.

36. Collins D, Winter DC. Modern concepts in diverticular disease. J Clin Gastroenterol. 2015;49(5):358-69.

37. Constantinides VA, Heriot A, Remzi F, Darzi A, Senapati A, Fazio VW, et al. Operative strategies for diverticular peritonitis: a decision analysis between primary resection and anastomosis vs. Hartmann's procedures. Ann Surg. 2007;245(1):94-103.

38. Strate LL, Peery AF, Neumann I. American Gastroenterological Association Institute technical review on the management of acute diverticulitis. Gastroenterology. 2015;149(7):1950-1976.e12.

39. Janes S, Meagher A, Frizelle FA. Elective surgery after acute diverticulitis. Br J Surg. 2005;92(2):133-42.

40. Thaler K, Weiss EG, Nogueras JJ, Arnaud JP, Wexner SD, Bergamaschi R. Recurrence rates at minimum 5-year follow-up: laparoscopic versus open sigmoid resection for uncomplicated diverticulitis. Surg Laparosc Endosc Percutan Tech. 2003;13(5): 325-7. 